\title{
Nomogram based on SIS predicting the survival of non-surgical thoracic esophageal squamous cell carcinoma treated by IMRT
}

\author{
Xingyu Du \\ The Fourth Hospital of Hebei Medical University \\ Shuchai Zhu ( $\nabla$ sczhu1965@163.com ) \\ The Fourth Hospital of Hebei Medical University \\ Jing Dong \\ The Fourth Hospital of Hebei Medical University \\ Ke Yan \\ The Fourth Hospital of Hebei Medical University \\ Xiaobin Wang \\ The Fourth Hospital of Hebei Medical University \\ Wenbin Shen \\ The Fourth Hospital of Hebei Medical University
}

\section{Research Article}

Keywords: esophageal squamous cell carcinoma, RT or CRT, systemic inflammation score, nomogram

Posted Date: April 16th, 2021

DOI: https://doi.org/10.21203/rs.3.rs-404753/v1

License: (-) (i) This work is licensed under a Creative Commons Attribution 4.0 International License. Read Full License 


\section{Abstract}

Background: Several inflammatory markers have been reported to be associated with clinical outcomes in patients with esophageal squamous cell carcinoma (ESCC). This study was to evaluate several preradiotherapy serum inflammatory indicators, including the neutrophil / lymphocytes ratio (NLR), platelet / lymphocyte (PLR), systemic immune-inflammatory index (SII), systemic inflammation score(SIS), and compare which one has the highest predicted survival value. Finally, combining inflammatory markers with traditional prognostic factors, a new Nomogram model was developed to predict overall survival (OS) and progression-free survival (PFS) for ESCC patients receiving radiotherapy (RT) or chemoradiotherapy (CRT).

Methods: This study retrospectively reviewed the data of 245 patients with thoracic esophageal squamous cell carcinoma (ESCC) underwent RT or CRT in the Fourth Hospital of Hebei Medical University from January 2013 to December 2015. The survival differences of these indexes were compared by the Kaplan-Meier method, and the univariate and the multivariate analyses were performed to determine these prognostic factors of overall survival (OS) and progression-free survival (PFS). Multivariate Cox proportional hazards regression models were used to create nomogram for OS and PFS.

Results: 239 patients met the eligibility criteria. The estimated 1-, 3-, and 5-year OS and PFS rates were 74.6\%, $36.8 \%, 26.5 \%$ and $58.4 \%, 31.3 \%, 20.5 \%$, respectively, for the whole group. The difference in survival between OS and PFS was significant when univariate analysis were applied based on these inflammation-based measures. Multivariate analysis showed that tumor length, T stage, TNM stage, chemotherapy, SIS were predictive variables for OS and PFS in the multivariate model. The nomogram model established based on multivariate models of training data set had good predictive ability, the unadjusted C-index was 0.701 ( $95 \%$ $\mathrm{Cl}, 0.662-0.740)$ and $0.695(95 \% \mathrm{Cl}, 0.656-0.734)$ for OS and PFS.

Conclusions: This study show that SIS, as a comprehensive indicator of inflammation and nutrition, had the strongest predictive power for evaluating prognosis. Moreover, our nomogram can accurately predict OS and PFS after treatment and may provide guidance regarding adjuvant therapy and surveillance.

\section{Background}

Esophageal cancer is a relatively common malignant disease, with 455800 newly diagnosed patients annually, and 400200 annual deaths from this disease[1]. Esophageal cancer is histologically classified into esophageal squamous cell carcinoma (ESCC), esophageal adenocarcinoma (EAC) and other subtypes. Historically, ESCC has been the dominant histological subtype, accounts for approximately $90 \%$ of esophageal cancers in eastern Asia, including China and Japan, and there is a trend of increasing incidence in some Asian countries. In China, esophageal squamous cell carcinoma accounts has been over than $90 \%$ [2]. Because most diagnoses were in advanced stages and clinical progress was rapid, $40 \% \sim 60 \%$ of patients lose the opportunity of surgery[3].

Due to lack of extensive prospective studies, it is difficult to accurately predict the prognosis of ESCC patients treated with the RT. Although several studies have reported that few parameters, such as the clinical symptoms and general state of the patients, tumour length, clinical staging, lymphatic/distant 
metastasis and squamous cell carcinoma-related antigen (SCC) levels, are related to the survival, they were not sufficiently powerful for evaluating the outcome. Therefore, a better predictive index for the survival time of patients with ESCC were importance and essential.

Relevant studies have proved that immune inflammation and nutritional indicators are closely related to the prognosis of many malignant tumors([4,5]. Potential prognostic values have been shown by changes in the levels of neutrophil, lymphocyte, platelet and monocyte. Meanwhile, various novel composite indicators have been calculated based on these peripheral blood parameters, including neutrophil / lymphocyte ratio (NLR), platelet / lymphocyte (PLR), lymphocyte / monocyte (LMR) and the composite index, for example, systemic immune-inflammatory index (SII), consisting neutrophil multiplied by the platelet divided by the lymphocyte, in some neoplasms[6]. In addition, There are several methods of assessing nutritional status in cancer patient of which serum albumin is one of the most commonly used[7]. However, there is no generally accepted optimal threshold for nutritional and inflammatory markers, and no combined inflammatory and nutritional indicators scoring system to incorporate these indicators in ESCC.

This study aims to evaluate the prognostic value of serum-based inflammatory and nutritional biomarkers for non-operative esophageal squamous cell carcinoma (ESCC), and to establish a comprehensive and innovative nomogram model for evaluating the prognosis of ESCC.

\section{Methods}

\section{Patients}

We retrospectively collected and analyzed pre-radiotherapy clinical and laboratory test data for 245 consecutive patients with thoracic ESCC who underwent radical RT or CRT at the Fourth Hospital of Hebei

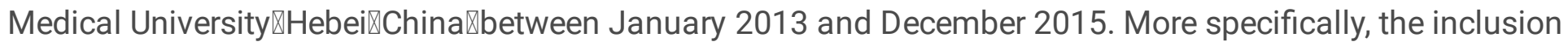
criteria were as follows: (1)Pathologically confirmed ESCC before RT, (2) Karnofsky Performance Score $\geq 70$ points, (4)patients refused surgery or did not undergo surgery for other reasons, (5)no history of malignant disease, and active double cancer at the time of diagnosis of esophageal cancer, (6)complete clinical and follow-up information was available. The exclusion criteria were (1) active hemorrhage or severe coagulation dysfunction, (2) severe uncontrolled hypertension, (3) severe cardiopulmonary diseases or abnormal liver and kidney function, (4) death from other diseases during follow-up, (5) incomplete data and (6) lost to follow-up.

\section{Data collection}

The limitation of the seventh edition of the 2009 AJCC TNM staging criteria is that it is applicable only to patients with surgical treatment alone, but not for patients with non-surgical. $n$ this study, the tumor staging before radiotherapy was classified according to the Clinical Classification of Esophageal Carcinoma Treated by Non-surgical Methods, the clinical prognostic value and practicability of which has been verified $[8,9]$.

All serum parameters were collected with in 7 days before RT, and including neutrophil, lymphocyte, monocyte, platelet counts, and albumin levels. NLR, LMR,PLR, and SII were graded by calculating the optimal cut-off values based on receiver operating characteristic (ROC) curves. NLR, PLR, LMR, SII, and SIS were calculated as follows NLR= neutrophil count/lymphocyte count, PLR=platelet count/lymphocyte count, 


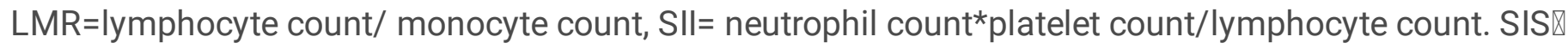
decreased serum albumin and decreased LMR ( $\leq 40 \mathrm{~g} / \mathrm{L}$ and $\leq 4.15$, respectively) were assigned SIS 2 , patients with either decreased serum albumin or decreased LMR were assigned SIS 1and patients with both elevated serum albumin and elevated $\mathrm{LMR}(>40 \mathrm{~g} / \mathrm{L}$ and $>4.15$, respectively) were assigned SIS 0 . All the patients signed informed consent forms, and all protocols in this study were approved by the ethics committee of the Fourth Hospital of Hebei Medical University.

\section{Follow-up}

According to our computer database patients were recorded and calculated to the date of death due to any cause. Patients who survived were examined in our survival analyses. Medical history, physical examination, laboratory examination, electrocardiogram, abdominal ultrasound, esophageal barium meal, and chest CT were tested every 3 months in the first two years and then every 6 months. Those who were lost to follow-up were cut off at the last follow-up time. Adverse events were assessed according to the National Cancer Institute's Common Terminology Criteria for Adverse Events (CTCAE v4.0).

\section{Radiotherapy}

Radiotherapy was performed according to standards published by the National Comprehensive Cancer Network (NCCN). In this study, 239 patients with ESCC received IMRT(95\% PTV / 50-66Gy / 25-33F, 1.8-2.0Gy / F, 5F / W). Imaging data from the computed tomography (CT) simulation scan were transmitted to the RT treatment planning system (Pinnacle, version 9.2, Philips Radiation Oncology System, USA), for delineation of the primary tumor area, enlargement lymph node and the organs at risk. The gross tumor volume (GTV), clinical tumor volume (CTV), and planned tumor volume (PTV) were outlined. The gross tumor volume (GTV) included esophagus with the thickened wall as shown by CT, esophageal film, and esophagoscopy and swollen lymph nodes with a short diameter $\geq 1 \mathrm{~cm}$. The clinical target volume (CTV) was $0.5 \mathrm{~cm}$ to $0.8 \mathrm{~cm}$ extended from GTV to the front, back, left, and right, and $2.5 \mathrm{~cm}$ to $3.0 \mathrm{~cm}$ extended on the top and bottom, plus the corresponding lymphatic drainage area. The planning target volume (PTV) was $0.6 \mathrm{~cm}$ extended from CTV on all sides. The treatment plan included the prescribed dose of 50-66Gy / 25-33F, 1.8-2.0Gy / F, 5F / Week, and the median dose of $61.2 \mathrm{~Gy}$. The total lungs V20 $\leq 30 \%$, V30 $\leq 20 \%$, V $5 \leq 60 \%$ and the lung mean dose $\leq 16 \mathrm{~Gy}$. The heart dose V25 $\leq 50 \%$, V40 $\leq 30 \%$ and the maximum Spinal Cord dose $<45 \mathrm{~Gy}$. The irradiated fields of esophageal lymph drainage were defined as CTV1. The scope of CTV1 varies according to the lesion location. The PTV1 was based on CTV1 Expand uniformly from 0.5 to $0.8 \mathrm{~cm}$ to the periphery. The required 95\% PTV1 prescription dose was 46 to 54 Gy, the 95\% PTV and 95\% PTV-nd prescription dose were 50 to $66 \mathrm{~Gy}, 1.80$ to 2.06 Gy per fraction, and 5 fractions per week.

\section{Chemotherapy}

According to NCCN for the concurrent chemoradiotherapy of Esophageal Cancer, three standard chemotherapy regimens were used in some patients: The first was cisplatin plus 5-fluorouracil (5-FU). Two cycles of cisplatin(75-100 mg/m2) on day 1 and 5-FU $\left(750-1000 \mathrm{mg} / \mathrm{m}^{2}\right)$ on day $1-4$, at an interval of 4 weeks were performed. As for maintenance chemotherapy, from approximately 4 weeks after CRT, two cycles 
of cisplatin $(75 \mathrm{mg} / \mathrm{m} 2)$ on day 1 and $5-\mathrm{FU}\left(750-1000 \mathrm{mg} / \mathrm{m}^{2}\right)$ on day $1-4$, One treatment cycle was 21 days, and the patients received 2 chemotherapy treatment cycles.

The second was docetaxel plus carboplatin. Docetaxel $(7.5 \mathrm{mg} / \mathrm{m} 2)$ on day 1 and carboplatin AUC=2 on day 1, One treatment cycle was 7 days, and the patients received 4-6 chemotherapy treatment cycles.

The third was docetaxel plus cisplatin. The dosage and administration schedule of docetaxel $(7.5 \mathrm{mg} / \mathrm{m} 2)$ intravenously on day 1 and continuous infusion of cisplatin $\left(75 \mathrm{mg} / \mathrm{m}^{2}\right)$ on day 1 . The chemotherapy treatment cycles were the same as for docetaxel plus carboplatin.

\section{Statistical Analysis}

The statistical analysis using Graphpad prism eversion 8.0 and R version 4.0.3 (R Foundation for Statistical Computing). Continuous variables were compared using the unpaired t test or Mann-Whitney nonparametric test. The chi-squared test and Fisher's exact test were used to compare the categorical variables. Survival analysis was conducted using Kaplan-Meier method and log-rank test. Multivariate analysis was performed using the Cox regression model for variables found to be significant among the univariate analysis, and the corresponding 95\% confidence intervals (Cls) were calculated, a two-tailed P-value $<0.05$ was considered statistically significant.

Receiver operating characteristic (ROC) curves were calculated to determine optimal cutoff values and to assess the predictive power of these inflammation-based indexes for long-term survival. Nomogram for 1 -, 3 and 5-year OS and PFS probabilities were constructed based on the multivariate Cox proportional hazards regression models. The discriminatory performance of the OS and PFS nomogram was evaluated by calculating Harrell's concordance index (C-index). In addition, we assessed calibration of the nomogram to compare the nomogram estimated risk to the observed risk. Calibration of the nomogram for 1-, 3- and 5years OS and PFS were illustrated in calibration plots.

\section{Results}

\section{Demographic and Clinical Characteristics}

239 patients met the eligibility criteria, 4 patients were excluded due to incomplete peripheral blood indicators, and 2 were excluded due to loss of follow-up. The survival sample numbers of 1, 3 and 5 years were 176, 83 and 62, respectively. The baseline characteristics of the patients were summarized in Table1. Their median age was 67 years (range, 41-90 years), There were 140 (58.6\%) male and 99 (41.4\%) female patients. The median tumor length was $6.2 \mathrm{~cm}$ (range, $2.35-15.9 \mathrm{~cm}$ ). The most common tumor location was middle thoracic lesion with 104 (43.5\%) patients. Upper thoracic lesion with 80(33.5\%) and lower thoracic lesion with

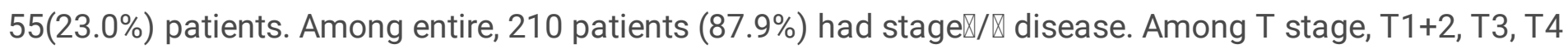
were 68(28.5\%), 83(34.7\%) and 88(36.8\%) respectively. 154 (64.4\%) had LN metastases, N0, N1, N2 were $85(35.6 \%), 109(45.6 \%)$ and $45(18.8 \%)$ respectively. Treatment modalities showed that, $110(46.0 \%)$ received IMRT alone and $129(54 \%)$ received CRT. Patients treated by IFI had 139(58.2) and ENI had 100(41.8\%). A total of $62(25.9 \%)$ patients had SIS 0, 115(48.1) of patients had SIS 1, and 62 (25.9\%) SIS2. Moreover, Gender, 
tumor location, tumor length, T stage, TNM stage, chemotherapy, RT modalities and prescription RT dose had statistical significance among different grades of SIS $(P<0.05$, Table 2$)$.

Table 1. Patient Characteristics of 239 Patients with ESCC

\begin{tabular}{|c|c|}
\hline Characteristics & Cases』numbers, \%ם \\
\hline Gender, male / female & $140 \square 58.6 \square / 99 \square 41.4 \square$ \\
\hline Age (years), $\leq 67 / 067$ & $127 \square 53.1 \square / 112 \square 46.9 \square$ \\
\hline Tumor location, upper / middle / lower thoracic & $80 \square 33.5 \square / 104 \square 43.5 \square / 55 \square 23.0 \square$ \\
\hline Tumor length $(\mathrm{cm}), \leq 6.2 / \square 6.2$ & $121 \square 50.6 \square / 118 \square 49.4 \square$ \\
\hline T stage, $1+2 / 3 / 4$ & $68(28.5) / 83 \square 34.7 \square / 88 \square 36.8 \square$ \\
\hline N stage, $0 / 1 / 2$ & $85 \square 35.6 \square / 109 \square 45.6 \square / 45 \square 18.8 \square$ \\
\hline TNM stage, $\square / \square / \square$ & $29 \square 12.1 \square / 97 \square 40.6 \square / 113 \square 47.3 \square$ \\
\hline CRT, no / yes & $110 \square 46.0 \square / 129 \square 54.0 \square$ \\
\hline Radiotherapy modalities, IFI / ENI & $139 \square 58.2 \square / 100 \square 41.8 \square$ \\
\hline prescription RT dose (Gy), $\leq 61.2 / \square 61.2$ & $135 \square 56.5 \square / 104 \llbracket 43.5 \square$ \\
\hline $\mathrm{NLR}, \leq 2.24 />2.24$ & 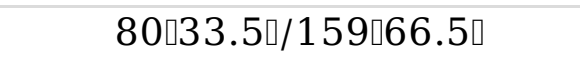 \\
\hline $\mathrm{PLR}, \leq 148 />148$ & $122 \square 51.0 \square / 117 \square 49.0 \square$ \\
\hline LMR, $\leq 4.15 />4.15$ & $155 \square 64.9 \square / 84 \square 35.1 \square$ \\
\hline Albumin, $\leq 40 />40$ & $152 \square 63.6 \square / 87 \square 36.4 \square$ \\
\hline SII, $\leq 738 />738$ & $144 \square 60.3 \square / 95 \square 39.7 \square$ \\
\hline SIS, 0 / $1 / 2$ & $62(25.9) / 115(48.2) / 62(25.9)$ \\
\hline
\end{tabular}

CRT : Chemoradiotherapy, IFI : Involved-field irradiation,ENI:Elective nodal irradiation, RT:Radiotherapy, NLR : Neutrophil-to-lymphocyte ratio, LMR: Lymphocyte-to-monocyte ratio, PLR : Platelet-to-lymphocyte, SII : Systemic immune-inflammatory index, SIS: Systemic inflammation score.

Table 2. Relationship between SIS before radiotherapy and clinicopathological characteristics of ESCC patients 


\begin{tabular}{|c|c|c|c|c|c|c|}
\hline \multirow[t]{2}{*}{ Characteristics } & \multirow[t]{2}{*}{ Parameter } & \multicolumn{3}{|c|}{ SIS, no.(\%) } & \multirow[t]{2}{*}{$\mathrm{X}^{2}$} & \multirow[t]{2}{*}{ p Value } \\
\hline & & 0 & 1 & 2 & & \\
\hline \multirow[t]{2}{*}{ Gender } & Male & $30 \square 12.6 \square$ & 77ロ32.2ם & 33प13.8ם & 6.713 & 0.035 \\
\hline & Female & $32 \square 13.4 \square$ & $38 \square 15.9 \square$ & $29 \square 12.1 \square$ & & \\
\hline \multirow[t]{2}{*}{ Age(years) } & $\leq 67$ & $34 \square 14.2 \square$ & $62 \square 25.9 \square$ & $31 \square 13.0 \square$ & 0.345 & 0.842 \\
\hline & $\square 67$ & 28ロ11.7ロ & $53 \square 22.2 \square$ & $31 \square 13.0 \square$ & & \\
\hline \multirow[t]{3}{*}{ Tumor location } & $\begin{array}{l}\text { Upper } \\
\text { thoracic }\end{array}$ & $33 \square 13.8 \square$ & $37 \square 15.5 \square$ & $10 \square 4.2 \square$ & 19.986 & 0.001 \\
\hline & $\begin{array}{l}\text { Middle } \\
\text { thoracic }\end{array}$ & $19 \square 7.9 \square$ & $53 \square 22.8 \square$ & $32 \square 13.4 \square$ & & \\
\hline & $\begin{array}{l}\text { Lower } \\
\text { thoracic }\end{array}$ & $10 \square 4.2 \square$ & $25 \square 10.5 \square$ & 20ロ8.4ロ & & \\
\hline \multirow[t]{2}{*}{ Tumor length median $\square \mathrm{cm} \square$} & $\leq 6.2$ & $46 \square 19.2 \square$ & $57 \square 23.8 \square$ & $18 \square 7.5 \square$ & 25.394 & $<0.001$ \\
\hline & $\square 6.2$ & $16 \square 6.7 \square$ & $58 \square 24.3 \square$ & $44 \square 18.4 \square$ & & \\
\hline \multirow[t]{3}{*}{ T stage } & $1+2$ & $27 \square 11.3 \square$ & $30 \square 12.6 \square$ & $11 \square 4.6 \square$ & 16.129 & 0.003 \\
\hline & 3 & 23ロ9.6ロ & $40 \square 16.7 \square$ & $20 \square 8.4 \square$ & & \\
\hline & 4 & $12 \square 5.0 \square$ & $45 \square 18.8 \square$ & $31 \square 13.0 \square$ & & \\
\hline \multirow[t]{3}{*}{$\mathrm{N}$ stage } & 0 & $26 \square 10.9 \square$ & $26 \square 10.9 \square$ & $10 \square 4.2 \square$ & 8.050 & 0.090 \\
\hline & 1 & $26 \square 10.9 \square$ & $62 \square 26.0 \square$ & $21 \square 8.9 \square$ & & \\
\hline & 2 & $10 \square 4.2 \square$ & $20 \square 8.4 \square$ & $15 \square 6.3 \square$ & & \\
\hline \multirow[t]{3}{*}{ TNM stage } & 口 & $14(5.9)$ & $13(5.4)$ & $2(1.4)$ & 15.200 & 0.004 \\
\hline & प & $27(11.3)$ & $48(20.1)$ & $22(9.2)$ & & \\
\hline & प & $21(8.8)$ & $54(22.6)$ & $38(15.9)$ & & \\
\hline \multirow[t]{2}{*}{ CRT } & N0 & $27(11.3)$ & $46(19.2)$ & $37(15.5)$ & 6.485 & 0.039 \\
\hline & Yes & $35 \square 14.6 \square$ & $69 \llbracket 28.9 \square$ & $25 \square 10.5 \square$ & & \\
\hline \multirow{3}{*}{$\begin{array}{l}\text { Radiotherapy } \\
\text { modalities }\end{array}$} & IFI & $27(11.3)$ & $62(25.9)$ & $50(20.9)$ & 19.173 & $<0.001$ \\
\hline & & & & & & \\
\hline & ENI & 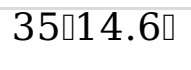 & 53ロ22.2\ & $12 \square 5.0 \square$ & & \\
\hline \multirow[t]{2}{*}{ prescription RT dose $\llbracket \mathrm{Gy} \square$} & $\leq 61.2$ & $33(13.8)$ & $58(24.3)$ & $44(18.4)$ & 7.271 & 0.026 \\
\hline & $\square 61.2$ & $29(12.1)$ & $57(23.8)$ & $18(7.5)$ & & \\
\hline
\end{tabular}

CRT : Chemoradiotherapy, RT:Radiotherapy.

\section{OS and prognostic factors}


All of patients the 1-,3-,5-year OS rates were $74.6 \%, 36.8 \%$ and $26.5 \%$, respectively. The median OS was 21.6 months (range, 1.7-95.3 months). The correlations between inflammation-based indexes and OS were shown in Fig. 1(A-D), elevated NLR $(P<0.05$, Fig. 1A), PLR $(P<0.05$, Fig. 1B), SII $(P<0.05$, Fig. $1 C)$ and SIS $(P$ $<0.05$, Fig. 1D) were associated with reduced OS. The univariate analysis revealed significant differences in tumor location, tumor length, T stage, N stage, TNM stage, chemotherapy, RT modalities, prescription RT dose, NLR, PLR, SII and SIS between better and poor outcome groups $(p<0.05$, Table 3$)$. The different grades of SIS were negative correlation with OS ( $p<0.001$, Fig. $1 \mathrm{D}$ ). Then, multivariate regression analysis was performed to identify parameters independently correlated associated with OS. Result revealed that SIS was an independent prognostic predictor of ESCC patients (HR 1.480, 95\% $(\mathrm{Cl}), 1.153-1.900, p=0.002)$. Other established predictors included tumor length $(p<0.001)$, T stage $(p=0.001)$, TNM stage $(p=0.003)$, and chemotherapy $(p=0.001)$, all were shown in Table 3 .

Table 3. Univariate and multivariate analyses of OS in patients with ESCC 


\begin{tabular}{|c|c|c|c|c|c|}
\hline \multirow[t]{2}{*}{ Characteristics } & & \multicolumn{2}{|c|}{ Univariate Analysis } & \multicolumn{2}{|c|}{ Multivariate Analysis } \\
\hline & & $\mathrm{X}^{2}$ & $\mathbf{P}$ & HR(95\% CI) & p Value \\
\hline Gender & male / female & 0.029 & 0.864 & & \\
\hline Age (years) & $\leq 67 / \square 67$ & 0.001 & 0.988 & & \\
\hline \multirow[t]{3}{*}{ Tumor location } & Upper thoracic & 7.520 & 0.023 & Reference & \\
\hline & Middle thoracic & & & $0.802 \square 0.532-1.208 \square$ & 0.291 \\
\hline & Lower thoracic & & & $1.028 \square 0.651-1.624 \square$ & 0.906 \\
\hline \multirow[t]{2}{*}{ Tumor length (cm) } & $\leq 6.2$ & 66.384 & $<0.001$ & Reference & \\
\hline & $\square 6.2$ & & & 1.699ロ1.168-2.470ロ & 0.006 \\
\hline \multirow[t]{3}{*}{ T stage } & $1+2$ & 19.983 & $<0.001$ & Reference & \\
\hline & 3 & & & $1.284 \square 0.830-1.987 \square$ & 0.262 \\
\hline & 4 & & & $0.513 \square 0.293-0.897 \square$ & 0.019 \\
\hline \multirow[t]{3}{*}{ N stage } & 0 & 15.130 & 0.001 & Reference & \\
\hline & 1 & & & 0.996ロ0.689-1.441ם & 0.984 \\
\hline & 2 & & & 1.098ロ0.649-1.857ロ & 0.729 \\
\hline \multirow[t]{3}{*}{ TNM stage } & I & 40.737 & $<0.001$ & Reference & \\
\hline & II & & & $1.884(0.923-3.846)$ & 0.082 \\
\hline & III & & & $4.422(1.948-10.038)$ & $<0.001$ \\
\hline \multirow[t]{2}{*}{ CRT } & No & 11.612 & $<0.001$ & Reference & \\
\hline & Yes & & & $0.669(0.485-0.923)$ & 0.014 \\
\hline Radiotherapy modalities & IFI & 11.615 & $<0.001$ & Reference & \\
\hline & ENI & & & $0.757(0.524-1.092)$ & 0.136 \\
\hline
\end{tabular}




prescription RT dose (Gy) $\quad \leq 61.2 \quad 4.254 \quad 0.039 \quad$ Reference

061.2

$0.978(0.702-1.363) \quad 0.896$

\begin{tabular}{|c|c|c|c|c|c|}
\hline NLR & $\leq 2.24$ & 7.170 & 0.007 & Reference & \\
\hline & $>2.24$ & & & $0.948(0.616-1.459)$ & 0.808 \\
\hline \multirow[t]{2}{*}{ PLR } & $\leq 148$ & 4.428 & 0.035 & Reference & \\
\hline & $>148$ & & & $1.170(0.814-1.681)$ & 0.397 \\
\hline \multirow[t]{2}{*}{ SII } & $\leq 738$ & 8.040 & 0.005 & Reference & \\
\hline & $>738$ & & & $1.111 \llbracket 0.743-1.662 \square$ & 0.609 \\
\hline \multirow[t]{3}{*}{ SIS } & 0 & 44.445 & $<0.001$ & Reference & \\
\hline & 1 & & & $1.937 \square 1.229-3.054 \square$ & 0.004 \\
\hline & 2 & & & $2.908 \square 1.678-5.041 \square$ & $<0.001$ \\
\hline
\end{tabular}

CRT : Chemoradiotherapy, IFI : Involved-field irradiation,ENI:Elective nodal irradiation, RT:Radiotherapy, NLR : Neutrophil-to-lymphocyte ratio, LMR: Lymphocyte-to-monocyte ratio, PLR : Platelet-to-lymphocyte, SII : Systemic immune-inflammatory index, SIS: Systemic inflammation score.

\section{PFS and prognostic factors}

The estimated 1-, 3-, and 5-year PFS rates with all patients were $58.4 \%, 31.3 \%$, and $20.5 \%$, respectively. The median PFS was 14.1 months (range, 1.3-95.3 months). The correlations between inflammation-based indexes and PFS were shown in Fig. 2. Elevated NLR ( $<<0.05$, Fig. $2 A)$, SII $(P<0.05$, Fig. $2 C)$ and $\operatorname{SIS}(P<$ 0.05 , Fig. 2D) were associated with reduced PFS. Nevertheless, PLR failed to distinguish patients with longer PFS from those shorter (Fig. 2B). Univariate survival analysis revealed significant association between unfavourable PFS and higher NLR, SII and SIS $(p<0.05$, Table 4). Other significant parameters related to PFS included tumor location, tumor length, T stage, N stage, TNM stage, chemotherapy, RT modalities, and prescription RT dose were administered $(p<0.05$, Table 4$)$. Multivariate Cox proportional hazards analysis showed that TNM stage (HR 1.538, 95\% Cl 1.045-2.266, P = 0.029), chemotherapy(HR 0.639, 95\% Cl 0.464$0.880, \mathrm{P}=0.006$ ) and SIS (HR 1.615, 95\% Cl 1.249-2.088, $\mathrm{P}<0.001$ ) were independent predictors of PFS (Table 4).

Table 4. Univariate and multivariate analyses of PFS in patients with ESCC 


\begin{tabular}{|c|c|c|c|c|c|}
\hline \multirow[t]{2}{*}{ Characteristics } & & \multicolumn{2}{|c|}{ Univariate Analysis } & \multicolumn{2}{|c|}{ Multivariate Analysis } \\
\hline & & $\mathrm{X}^{2}$ & $\mathrm{P}$ & $\mathrm{HR}(95 \% \mathrm{CI})$ & $\mathrm{P}$ \\
\hline Gender & male / female & 0.004 & 0.947 & & \\
\hline Age (years) & $\leq 67 / \square 67$ & 0.005 & 0.946 & & \\
\hline \multirow[t]{3}{*}{ Tumor location } & Upper thoracic & 12.071 & 0.002 & Reference & \\
\hline & Middle thoracic & & & $0.750 \square 0.485-1.161 \square$ & 0.197 \\
\hline & Lower thoracic & & & $1.031 \square 0.623-1.708 \square$ & 0.905 \\
\hline \multirow[t]{2}{*}{ Tumor length (cm) } & $\leq 6.2$ & 48.696 & $<0.001$ & Reference & \\
\hline & $\square 6.2$ & & & $1.534 \square 1.029-2.285 \square$ & 0.036 \\
\hline \multirow[t]{3}{*}{ T stage } & $1+2$ & 15.424 & $<0.001$ & Reference & \\
\hline & 3 & & & $1.111 \llbracket 0.709-1.740 \square$ & 0.645 \\
\hline & 4 & & & $0.557 \square 0.307-11.011 \square$ & 0.054 \\
\hline \multirow[t]{3}{*}{$\mathrm{N}$ stage } & 0 & 14.883 & 0.001 & Reference & \\
\hline & 1 & & & $1.014 \llbracket 0.690-1.489 \square$ & 0.944 \\
\hline & 2 & & & $1.349 \square 0.758-2.399 \square$ & 0.309 \\
\hline \multirow[t]{3}{*}{ TNM stage } & I & 31.714 & $<0.001$ & Reference & \\
\hline & II & & & $2.065 \square 0.998-4.275 \square$ & 0.050 \\
\hline & III & & & $3.665 \square 1.574-8.685 \square$ & 0.003 \\
\hline \multirow[t]{2}{*}{ CRT } & No & 10.174 & 0.001 & Reference & \\
\hline & Yes & & & $0.651 \llbracket 0.466-0.909 \square$ & 0.012 \\
\hline Radiotherapy modalities & IFI & 15.653 & $<0.001$ & Reference & \\
\hline & ENI & & & 0.745ロ0.507-1.095ロ & 0.135 \\
\hline
\end{tabular}




\begin{tabular}{|c|c|c|c|c|c|}
\hline prescription RT dose (Gy) & $\leq 61.2$ & 7540 & 0.006 & Reference & \\
\hline & $\square 61.2$ & & & $0.908 \square 0.636-1.296 \square$ & 0.594 \\
\hline \multirow[t]{2}{*}{ NLR } & $\leq 2.24$ & 7.125 & 0.008 & Reference & \\
\hline & $>2.24$ & & & 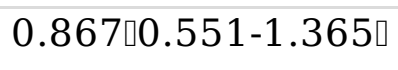 & 0.583 \\
\hline PLR & $\leq 148 />148$ & 2.606 & 0.106 & & \\
\hline \multirow[t]{2}{*}{ SII } & $\leq 738$ & 13.040 & $<0.001$ & Reference & \\
\hline & $>738$ & & & $1.337 \square 0.883-2.024 \square$ & 0.170 \\
\hline \multirow[t]{3}{*}{ SIS } & 0 & 50.747 & $<0.001$ & Reference & \\
\hline & 1 & & & $2.689 \square 1.648-4.388 \square$ & $<0.001$ \\
\hline & 2 & & & 3.562ロ1.992-6.368ם & $<0.001$ \\
\hline
\end{tabular}

CRT : Chemoradiotherapy, IFI : Involved-field irradiation,ENI:Elective nodal irradiation, RT:Radiotherapy, NLR : Neutrophil-to-lymphocyte ratio, LMR: Lymphocyte-to-monocyte ratio, PLR : Platelet-to-lymphocyte, SII : Systemic immune-inflammatory index, SIS: Systemic inflammation score.

\section{Predictive value of inflammatory indexes}

The ROC curve analysis was performed to estimate predictive powerful of the inflammation based indexes for both OS and PFS in ESCC patients. The ROC curves for OS and PFS prediction were calculated with patient's 5 years of follow-up. More specifically, results showed the AUROC values of SIS were consistently higher than other inflammatory index with area under curve (AUC) of 0.731 for OS and (AUC) of 0.755 for PFS. Subsequently, A comparative study revealed that patients with higher SIS occurred unfavorable outcome more frequently, and they had statistical significance among different grades of SIS in 1,3,5 OS and PFS (Fig. 3) (Table 5).

Table 5. The comparison of the difference SIS effect on OS and PFS 


\begin{tabular}{|c|c|c|c|c|c|c|c|c|c|c|}
\hline \multirow[t]{2}{*}{ Time } & \multicolumn{3}{|c|}{ OS (number, \%) } & \multirow[t]{2}{*}{$\mathrm{X}^{2}$} & \multirow[t]{2}{*}{$P$} & \multicolumn{3}{|c|}{ PFS (number, \%) } & \multirow[t]{2}{*}{$\mathrm{X}^{2}$} & \multirow[t]{2}{*}{$P$} \\
\hline & $\begin{array}{c}0 \square \\
(n=62)\end{array}$ & $\begin{array}{c}1 \square \\
\square n=115 \square\end{array}$ & $\begin{array}{c}2 \square \\
\square \mathrm{n}=62 \square\end{array}$ & & & $\begin{array}{c}0 \square \\
\square n=62 \square\end{array}$ & $\begin{array}{c}1 \square \\
\square n=115 \square\end{array}$ & $\begin{array}{c}2 \square \\
\square n=62 \square\end{array}$ & & \\
\hline $1 \mathrm{y}$ & $\begin{array}{c}55 \\
\square 88.7 \square\end{array}$ & $\begin{array}{c}89 \\
\square 77.4 \square\end{array}$ & $\begin{array}{c}32 \\
051.6 \square\end{array}$ & 23.58 & $<0.0001$ & $\begin{array}{c}48 \\
\square 77.4 \square\end{array}$ & $\begin{array}{c}61 \\
\square 53.0 \square\end{array}$ & $\begin{array}{c}21 \\
\square 33.9 \square\end{array}$ & 23.86 & $<0.0001$ \\
\hline $3 y$ & $\begin{array}{c}35 \\
056.5 \square\end{array}$ & $\begin{array}{c}40 \\
\square 34.8 \square\end{array}$ & $\begin{array}{c}8 \\
\square 12.9 \square\end{array}$ & 25.94 & $<0.0001$ & $\begin{array}{c}31 \\
050.0 \square\end{array}$ & $\begin{array}{c}23 \\
\square 20.0 \square\end{array}$ & $3 \square 4.8 \square$ & 36.62 & $<0.0001$ \\
\hline $5 y$ & $\begin{array}{c}30 \\
\square 48.4 \square\end{array}$ & $\begin{array}{c}27 \\
\square 23.5 \square\end{array}$ & $5 \square 8.0 \square$ & 26.94 & $<0.0001$ & $\begin{array}{c}26 \\
\square 41.9 \square\end{array}$ & $\begin{array}{c}21 \\
\square 18.3 \square\end{array}$ & $2 \square 3.2 \square$ & 29.18 & $<0.0001$ \\
\hline
\end{tabular}

\section{Established and assessed the nomogram model}

To better visualize the risk point and predictive power for OS and PFS in patients with ESCC, we constructed a prognostic nomogram based on multivariate cox regression analysis results, including tumor length, TNM stage, chemotherapy and SIS (Figure 4,5). The total scores of these prognostic factors can be used to determine the probabilities of 1-3- and 5-years OS and PFS. The performance of the nomogram were evaluated using the C-index and calibration curves. The C-index for OS prediction was 0.701 ( $95 \% \mathrm{Cl}$ : $0.662-$ 0.740 ) and PFS prediction was 0.695 (95\% Cl:0.656-0.734). The calibration graph showed properly probability consistencies between standard curve and nomogram prediction. The calibration curves also had good prediction consistency (Figures 6).

\section{Discussion}

Recent studies showed that several inflammation factors including NLR, PLR, LMR and SII, etc. were associated with long time prognosis survival for esophageal squamous cell carcinoma patients[10,11, 12]. Although most studies have demonstrated the prognostic relationship between related inflammatory markers in esophageal squamous cell carcinoma, no comparison of prognostic value has been conducted, nor has a comprehensive prognostic model been developed. Therefore Inflammatory markers were assessed and rigorous statistical methods were required. Besides, the nomogram model based on traditional prognostic indicators and SIS considered as a trustworthy method to generate more accurate prediction of prognosis.

In this study, We investigated clinical characteristics and prognosis of 239 ESCC patients treated by RT or CRT with periphery blood markers of systemic inflammation and nutrition parameters. We systematically investigated clinical characteristics and demonstrated the prognostic significance of pre-radiotherapy inflammatory parameters, including NLR, PLR, SII and the composite index SIS based on the level of lymphocyte-to-monocyte ratio (LMR) and serum albumin (Alb) for predicting survival status. Studies have found that SIS has a high prognostic value in patients with ESCC and is a excellent supplement to the traditional prognostic indicators.

Our studies have shown that patients with later lesion, such as tumor length $>6.2 \mathrm{~cm}$, TNM stage III showed a significant increase in SIS1-2. On the contrary, the number of SIS0-1 in patients with tumor length $\leq 6.2 \mathrm{~cm}$ and early TNM stage is more. This indicates that with the progression of the disease, the immune 
inflammatory response of the patients is gradually aggravated. In addition, albumin levels are reduced in patients with late stage due to malnutrition, anorexia or abnormal liver function. So people with high SIS scores tend to have a poor prognosis. In terms of treatment methods, patients with SIS0-1 preferred combined chemotherapy, ENI and prescription RT dose $>61.2$, while SIS2 preferred RT alone, IFI and RT dose $\leq 61.2$. This suggests that patients with a lower SIS score who can tolerate more thorough treatment tend to have a better prognosis.

The prognostic value of SIS in predicting malignant tumors has been reported. Y chang, et al. establish the SIS defined as follows: patients with both decreased serum albumin and decreased LMR ( $<40 \mathrm{~g} / \mathrm{L}$ and $<4.44$, respectively) were assigned SIS 2, patients with either decreased serum albumin or decreased LMR were assigned SIS 1 and patients with both elevated serum albumin and elevated LMR( $\geq 40 \mathrm{~g} / \mathrm{L} 1$ and $\geq 4.44$, respectively) were assigned SIS 0 . Their final results showed that SIS was an independent prognostic factors of OS in renal cell carcinoma. High SIS was associated with shorter OS[13]. Shoichi Inokuchi, et al. Using ROC curves showed SIS is a good predictor of OS and RFS for patients with hepatocellular carcinoma.

The same results were obtained in our study, the lower the SIS, the higher the survival. Multivariate analysis showed that SIS was an independent prognostic factor for OS and PFS with ESCC patients.In our study, multivariate analysis showed that SIS closely correlated with clinical characteristics and was an independent prognostic factor for OS and PFS in ESCC patients treated by RT or CRT. According to the area under the ROC curve, SIS was found to had higher predictive value than other indicators. The SIS AUC of OS and PFS higher than other inflammatory factors were 0.731 and 0.755 , respectively.

However, though the prognostic value of SIS has been shown for predicting the survival of patients with ESCC, the mechanism is still unclear. A lower of SIS indicates low level of LMR and/or albumin, which might increased nonspecific inflammation, and immune system dysfunction, and malnutrition.

For one thing, the probable reason for low level of LMR is that increased monocytes and/or reduced lymphocytes. Monocytes are known to promote tumorigenesis and angiogenesis through local immune suppression and stimulation of tumor neovasculogenesis [14]. Moreover, tumor-associated macrophages developing from mono nuclear cell lineages have been demonstrated to be able to inhibit cancer progression and spread of tumors[15]. This could explain why an elevated monocyte counts to confer poor clinical outcomes of various types of cancers. Noriyuki,H, et al. reported LMR was associated with cancer-specific survival (CSS) of esophageal cancer patients after curative esophagectomy. In particular, a low LMR was an independent predictor of poor survival in non-elderly patients[16].

There is increasing evidence that lymphocytes are essential for antitumor immune reactions owing to several mechanisms, including the ability to enhance tumor cell apoptosis, inhibition of tumor cell proliferation, and promotion of metastasis[17]. Davuluri, Rajayogesh et al. demonstrated that Lymphocyte reduction during CRT for EC was associated with poor outcomes, suggesting a role of host immunity in disease control[18]. The same Zhang, Jian. also confirmed that lymphatic invasion, is an independent favorable prognostic factor of DFS in patients with NSCLC who underwent lobectomy and lymph node dissection and adjuvant chemotherapy[19]. These may explain the reason that low level of LMR may serve as an independent prognostic factor for OS and PFS. 
For another, serum albumin is generally used to assess the nutritional status, severity of disease, disease progression and as an independent prognosticator of survival in various cancers [20]. Malnutrition is closely related to the imbalance of tumor microenvironment. It weaken patient's defense mechanisms such as humoral immunity, cellular immunity and phagocytosis and resulting in increased risk of infection and poor efficacy of anti-tumor treatment[21]. Because nutrition is an important determinant of immune response, decreased serum albumin reflects both malnutrition and sustained systemic inflammation response [22]. Thus, SIS, which is based on LMR and serum albumin level, may predict prognostic as assessments for cancer. This observation provides a rationale to prospectively test chemotherapeutic and radiation treatment strategies that may have a lower impact on host immunity.

Finally, we endued scores based on the weight of each predictor and established a nomogram model[23, 24], aimed to better visualize the risk point and predictive power of SIS. Statistical analysis showed that this model had a good fitness of data and accuracy in predicting functional outcome. For example, one patient had tumor length $=5 \mathrm{~cm}$, TNM stage (II), combined chemotherapy, and SIS 2 . For this example, the OS nomogram model total point equals 164 , and the 1-, 3-, 5-years OS is approximately $81 \%, 42 \%$ and $29 \%$. The PFS nomogram model point equals 162 , and the $1-, 3-, 5$-years PFS is $59 \%, 24 \%$ and $18 \%$. This nomogram model show that the SIS can provide additional prognostic information to traditional prognostic factors and will be helpful assist clinicians to better optimized care and stratify patients of health care resources to improve ESCC patients prognosis. Meanwhile, it including the conventional prognostic factors and SIS can further improve the predictive ability, and helps to identify OS and PFS rate early. In other words, SIS had an high predictive value for ESCC patients,independent of conventional risk factors.

The advantage of this study is that SIS is superior to NLR, PLR and SII, and nomogram based on SIS could be a new prognostic scoring system model contributes to the risk assessment for patients with ESCC. What's more, the SIS measurements are easy to obtain and low cost because they are based on standard laboratory tests for serum albumin, lymphocyte, and monocyte counts used routinely in clinical practice.

Nonetheless there are a few limitations in present study. Firstly, this study as a retrospective study, a singlecenter design with the number of patients enrolled in the model was relatively small and no external validation. Secondly, this study did not further compare the dynamic change of peripheral blood inflammatory markers during and after RT. Therefore, a prospective verification trials with a great quantity of cases are needed to confirm our present findings.

\section{Conclusion}

In conclusion, the predictive power of several inflammation-based prognostic values were assessed and compared in patients with ESCC. The SIS were independently associated with OS and PFS and the nomogram model based on SIS had more favorable predictive ability. Patients with SIS 0 have better OS and PFS, and those with SIS 1 and SIS 2 should receive more better monitoring and more rigorous treatment to avoid tumor progression. However, further studies are necessary to validate this findings and explore the mechanisms, to provide evidence for individualized treatment of ESCC patients.

\section{Abbreviations}


ESCC: Esophageal squamous cell carcinoma, CRT: Chemoradiotherapy, IFI: Involved-field irradiation, ENI:Elective nodal irradiation, OS: Overall survival, PFS: Progression-free survival, RT:Radiotherapy, NLR : Neutrophil-to-lymphocyte ratio, LMR: Lymphocyte-to-monocyte ratio, PLR : Platelet-to-lymphocyte, SII : Systemic immune-inflammatory index, SIS: Systemic inflammation score.

\section{Declarations}

\section{Acknowledgements}

Not applicable.

\section{Authors' contributions}

$X Y D, S C Z$ conceived of and designed the study. J D, KY, XB W and WB S generated the figures and Tables. $X Y D$ and $J D$ analyzed the data. XY D wrote the manuscript and SC Z critically reviewed the manuscript. All authors have read and approved the final manuscript.

\section{Funding}

We received no external funding for this study.

\section{Availability of data and materials}

The datasets used and/or analysed during the current study are available from the corresponding author on reasonable request.

\section{Ethics approval and consent to participate}

The study was approved by the ethics committee of The Fourth Hospital of Hebei Medical University. Informed consent was obtained from all patients.

\section{Consent for publication}

Not applicable.

\section{Competing interests}

The authors disclose no conflicts of interest.

\section{Author details}

${ }^{1}$ Department of Radiation Oncology, The Fourth Hospital of Hebei Medical University, 12 Jiankang Road, Shijiazhuang, Hebei 050011.

\section{References}


[1]Bray F, Ferlay J, Soerjomataram I, Siegel RL, Torre LA, Jemal A. Global cancer statistics 2018: GLOBOCAN estimates of incidence and mortality worldwide for 36 cancers in 185 countries. CA Cancer J Clin. 2018 Nov;68(6):394-424. doi: 10.3322/caac.21492. Epub 2018 Sep 12. Erratum in: CA Cancer J Clin. 2020 Jul;70(4):313. PMID: 30207593.

[2]Torre LA, Bray F, Siegel RL, Ferlay J, Lortet-Tieulent J, Jemal A. Global cancer statistics, 2012. CA Cancer J Clin. 2015 Mar;65(2):87-108. doi: 10.3322/caac.21262. Epub 2015 Feb 4. PMID: 25651787.

[3]Hirano $\mathrm{H}$, Boku $\mathrm{N}$. The current status of multimodality treatment for unresectable locally advanced esophageal squamous cell carcinoma. Asia Pac J Clin Oncol. 2018 Aug;14(4):291-299. doi: 10.1111/ajco.12995. Epub 2018 Jun 5. PMID: 29873183.

[4]Kubo T, Ono S, Ueno H, Shinto E, Yamamoto J, Hase K. Impact of the perioperative neutrophil-tolymphocyte ratio on the long-term survival following an elective resection of colorectal carcinoma. Int $J$ Colorectal Dis. 2014 Sep;29(9):1091-9. doi: 10.1007 / s00384 -014 -1 964-1.Epub 2014 Jul 22. PMID: 25048875.

[5]Coussens LM, Werb Z. Inflammation and cancer. Nature. 2002 Dec 19-26;420(6917):860-7. doi: 10.1038/nature01322. PMID: 12490959; PMCID: PMC2803035.

[6]Kühn T, Sookthai D, Graf ME, Schübel R, Freisling H, Johnson T, Katzke V, Kaaks R. Albumin, bilirubin, uric acid and cancer risk: results from a prospective population-based study. $\mathrm{Br} \mathrm{J}$ Cancer. $2017 \mathrm{Nov}$ 7;117(10):1572-1579. doi: 10.1038/bjc.2017.313. Epub 2017 Sep 12. PMID: 28898231; PMCID: PMC5680462.

[7]McMillan DC. Systemic inflammation, nutritional status and survival in patients with cancer. Curr Opin Clin Nutr Metab Care. 2009 May;12(3):223-6. doi: 10.1097/MC0.0b013e32832a7902. PMID: 19318937.

[8]Zhi X, Jiang K, Shen Y, Su X, Wang K, Ma Y, Zhou L. Peripheral blood cell count ratios are predictive biomarkers of clinical response and prognosis for non-surgical esophageal squamous cell carcinoma patients treated with radiotherapy. J Clin Lab Anal. 2020 Oct;34(10):e23468. doi: 10.1002/jcla.23468. Epub 2020 Jul 17. PMID: 32681567; PMCID: PMC7595892.

[9]Qingshan Z, Jing S, Nengchao W, Zhijie H, Qingfu Z, Lianjie F. Study of clinical staging of esophageal cancer treated by non-surgical methods. Can Res Prevent Treat. 2013;40(2):168-171.

[10]Gao Y, Guo W, Cai S, Zhang F, Shao F, Zhang G, Liu T, Tan F, Li N, Xue Q, Gao S, He J. Systemic immuneinflammation index (SII) is useful to predict survival outcomes in patients with surgically resected esophageal squamous cell carcinoma. J Cancer. 2019 Jun 2;10(14):3188-3196. doi: 10.7150/jca.30281. PMID: 31289589; PMCID: PMC6603384.

[11]Shao Y, Ning Z, Chen J, Geng Y, Gu W, Huang J, Pei H, Shen Y, Jiang J. Prognostic nomogram integrated systemic inflammation score for patients with esophageal squamous cell carcinoma undergoing radical esophagectomy. Sci Rep. 2015 Dec 22;5:18811. doi: 10.1038/srep18811. PMID: 26689680; PMCID: PMC4686940. 
[12]Liu JS, Huang Y, Yang X, Feng JF. A nomogram to predict prognostic values of various inflammatory biomarkers in patients with esophageal squamous cell carcinoma. Am J Cancer Res. 2015 Jun 15;5(7):21809. PMID: 26328248; PMCID: PMC4548329.

[13]Chang Y, An H, Xu L, Zhu Y, Yang Y, Lin Z, Xu J. Systemic inflammation score predicts postoperative prognosis of patients with clear-cell renal cell carcinoma. Br J Cancer. 2015 Aug 11;113(4):626-33. doi: 10.1038/bjc.2015.241. Epub 2015 Jul 2. PMID: 26135896; PMCID: PMC4647686.

[14]Koh YW, Kang HJ, Park C, Yoon DH, Kim S, Suh C, Go H, Kim JE, Kim CW, Huh J. The ratio of the absolute lymphocyte count to the absolute monocyte count is associated with prognosis in Hodgkin's lymphoma: correlation with tumor-associated macrophages. Oncologist. 2012;17(6):871-80. doi: 10.1634/theoncologist.2012-0034. Epub 2012 May 15. PMID: 22588324; PMCID: PMC3380887.

[15]Condeelis J, Pollard JW. Macrophages: obligate partners for tumor cell migration, invasion, and metastasis. Cell. 2006 Jan 27;124(2):263-6. doi: 10.1016/j.cell.2006.01.007. PMID: 16439202.

[16]Li Y, Liu W, Zhou Z, Ge H, Zhao L, Liu H, Song X, Wang D, Pei Q, Tan F. Development and validation of prognostic nomograms for early-onset locally advanced colon cancer. Aging (Albany NY). 2020 Dec 3;13(1):477-492. doi: 10.18632/aging.202157. Epub 2020 Dec 3. PMID: 33289705; PMCID: PMC7834989.

[17]Fridman WH, Pagès F, Sautès-Fridman C, Galon J. The immune contexture in human tumours: impact on clinical outcome. Nat Rev Cancer. 2012 Mar 15;12(4):298-306. doi: 10.1038/nrc3245. PMID: 22419253.

[18]Davuluri R, Jiang W, Fang P, Xu C, Komaki R, Gomez DR, Welsh J, Cox JD, Crane CH, Hsu CC, Lin SH. Lymphocyte Nadir and Esophageal Cancer Survival Outcomes After Chemoradiation Therapy. Int J Radiat Oncol Biol Phys. 2017 Sep 1;99(1):128-135. doi: 10.1016/j.jjrobp.2017.05.037. Epub 2017 Jun 1. PMID: 28816138.

[19] Kobayashi N, Usui S, Kikuchi S, Goto Y, Sakai M, Onizuka M, Sato Y. Preoperative lymphocyte count is an independent prognostic factor in node-negative non-small cell lung cancer. Lung Cancer. 2012 Feb;75(2):2237. doi: 10.1016/j.lungcan.2011.06.009. Epub 2011 Jul 20. PMID: 21764477.

[20]Maltoni M, Amadori D. Prognosis in advanced cancer. Hematol Oncol Clin North Am. 2002 Jun;16(3):71529. doi: 10.1016/s0889-8588(02)00024-2. PMID: 12170577.

[21]Arroyo V, García-Martinez R, Salvatella X. Human serum albumin, systemic inflammation, and cirrhosis. J Hepatol. 2014 Aug;61(2):396-407. doi: 10.1016/j.jhep.2014.04.012. Epub 2014 Apr 18. PMID: 24751830.

[22]McMillan DC. Systemic inflammation, nutritional status and survival in patients with cancer. Curr Opin Clin Nutr Metab Care. 2009 May;12(3):223-6. doi: 10.1097/MC0.0b013e32832a7902. PMID: 19318937.

[23]Ohori Tatsuo Gondo And Riu Hamada M, Gondo T, Hamada R. [Nomogram as predictive model in clinical practice]. Gan To Kagaku Ryoho. 2009 Jun;36(6):901-6. Japanese. PMID: 19542708. 
[24]Balachandran VP, Gonen M, Smith JJ, DeMatteo RP. Nomograms in oncology: more than meets the eye. Lancet Oncol. 2015 Apr;16(4):e173-80. doi: 10.1016/S1470-2045(14)71116-7. PMID: 25846097; PMCID:

PMC4465353.

\section{Figures}

A

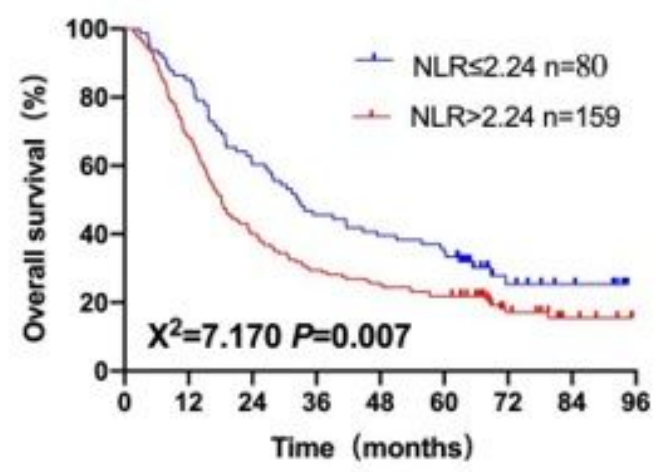

C

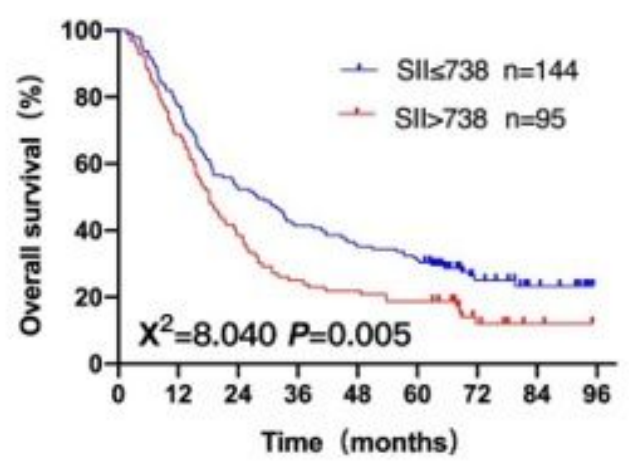

B

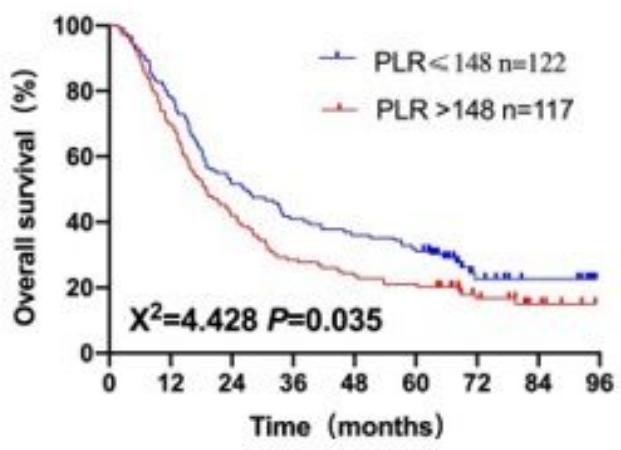

D

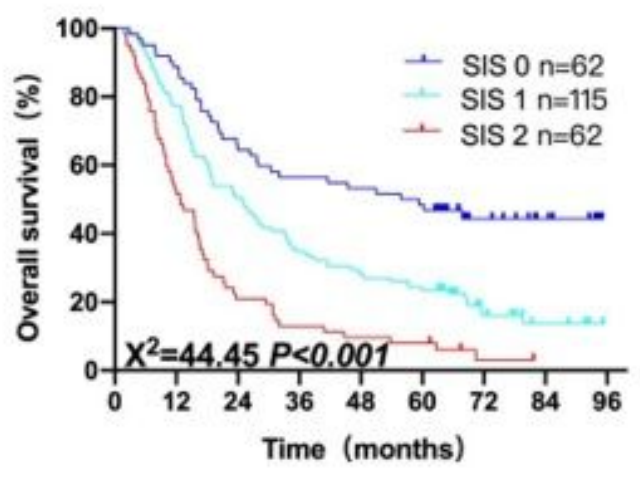

Figure 1

Kaplan-Meier survival curves of OS for different models: (A) NLR, (B) PLR (C) SII. (D) SIS. 

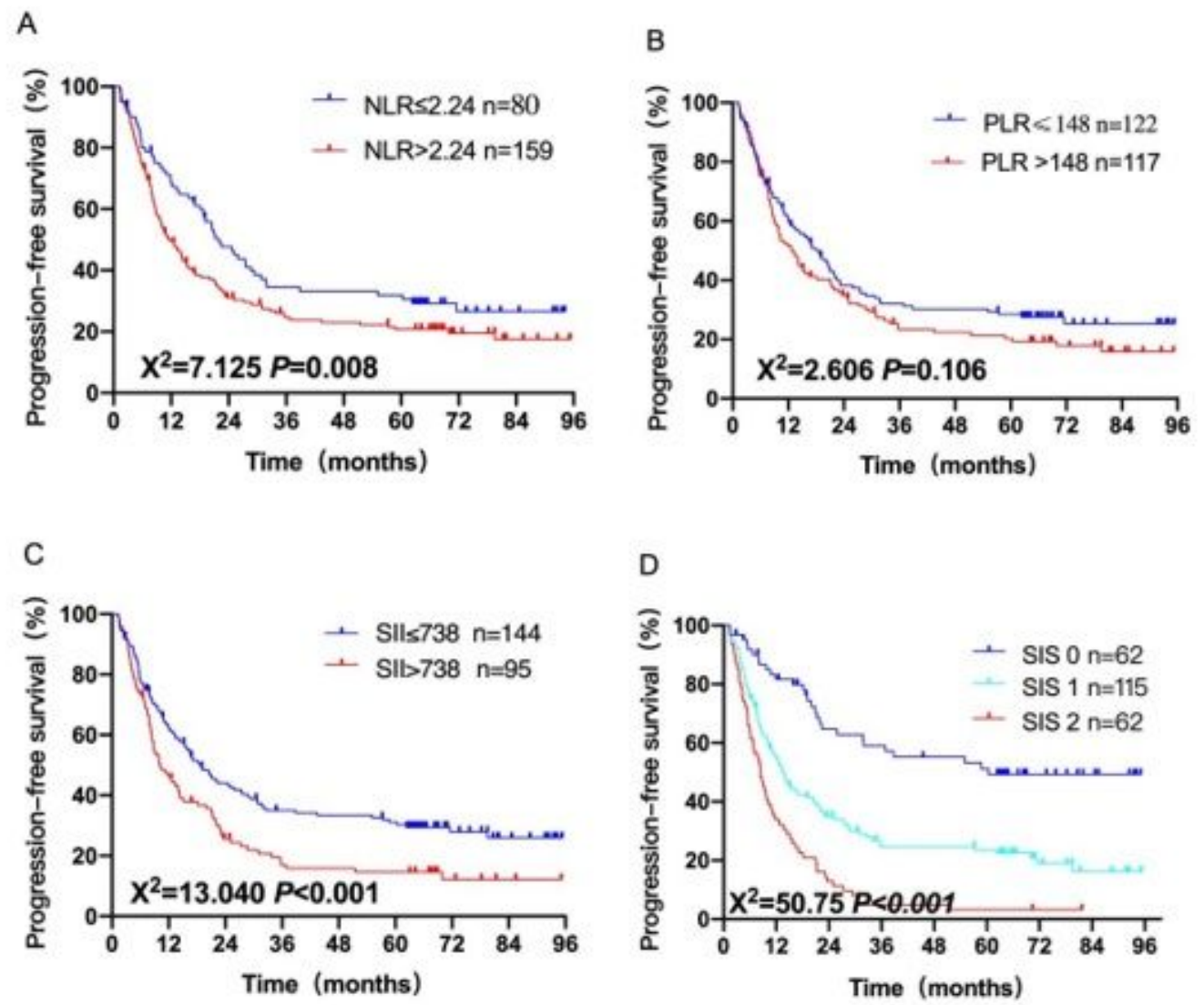

\section{Figure 2}

Kaplan-Meier survival curves of PFS for different models: (A) NLR, (B) PLR (C) SII. (D) SIS.
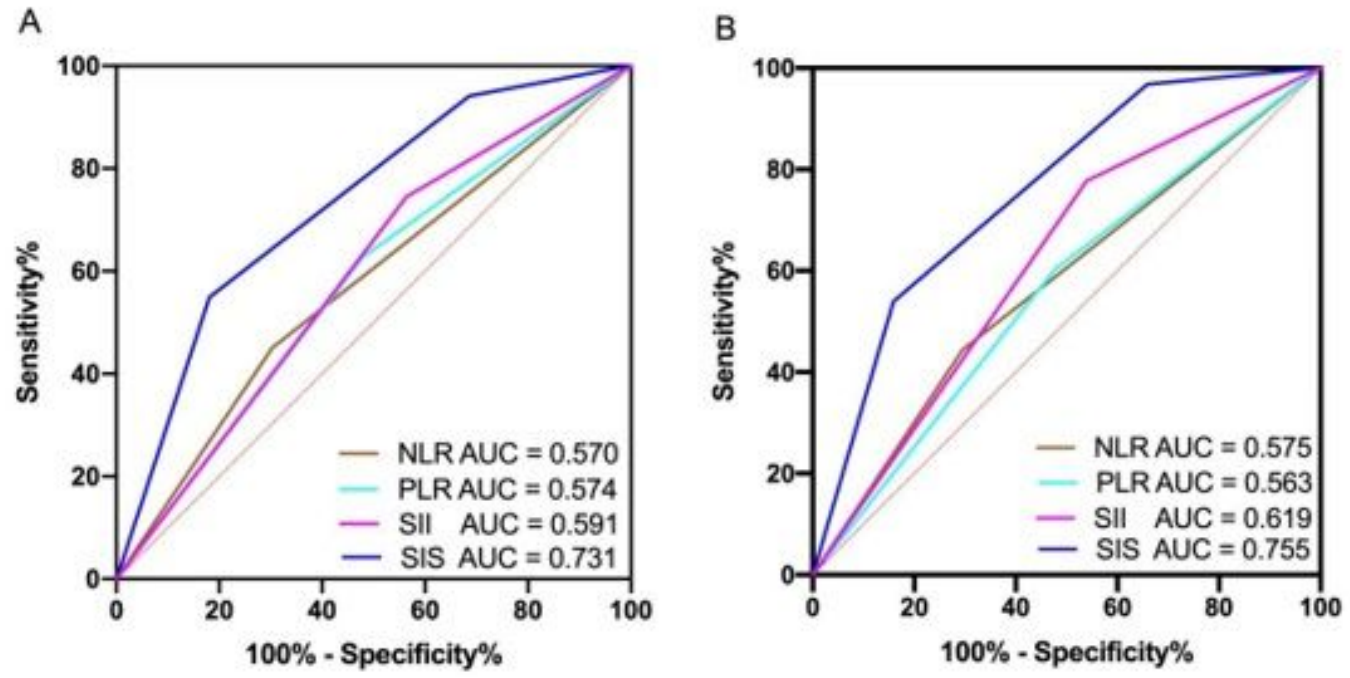

\section{Figure 3}

AUROC for OS (A) and PFS (B) stratifed by each inflammation-based index at 1-year, 3-year and 5-year. 

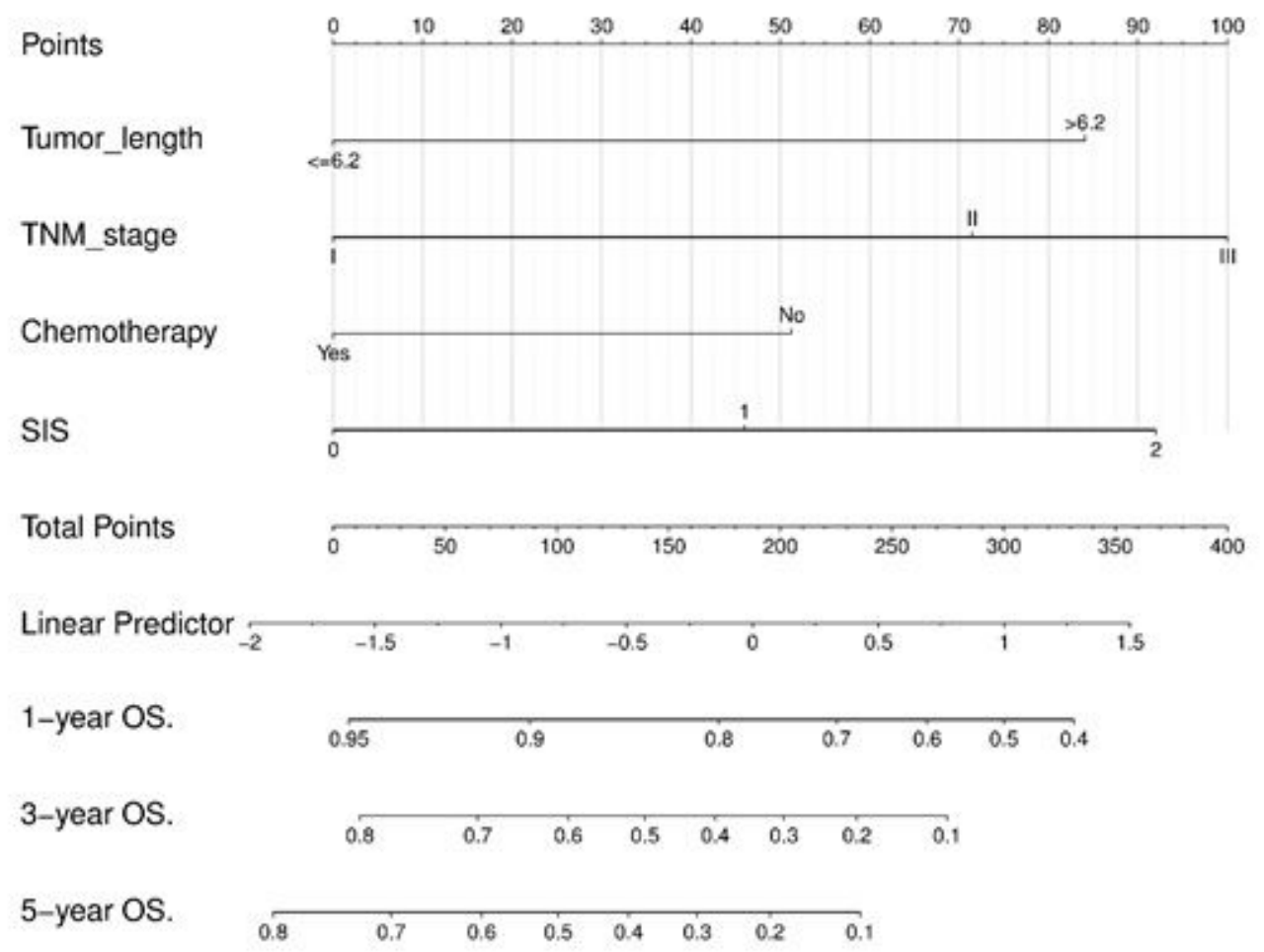

\section{Figure 4}

Nomogram predicting 1-, 3- and 5-year OS probabilities of patients with ESCC. Each risk factor was assigned a point according to the nomogram. The exact values of each factor are Tumor length ( 0,84 points), TNM stage ( 0,100 points), Chemotherapy ( 0,51 points), SIS ( 0,92 points). The total points were obtained by adding the points of all risk factors. Then, the 1-, 3- and 5-year OS could be predicted based on the total points. 

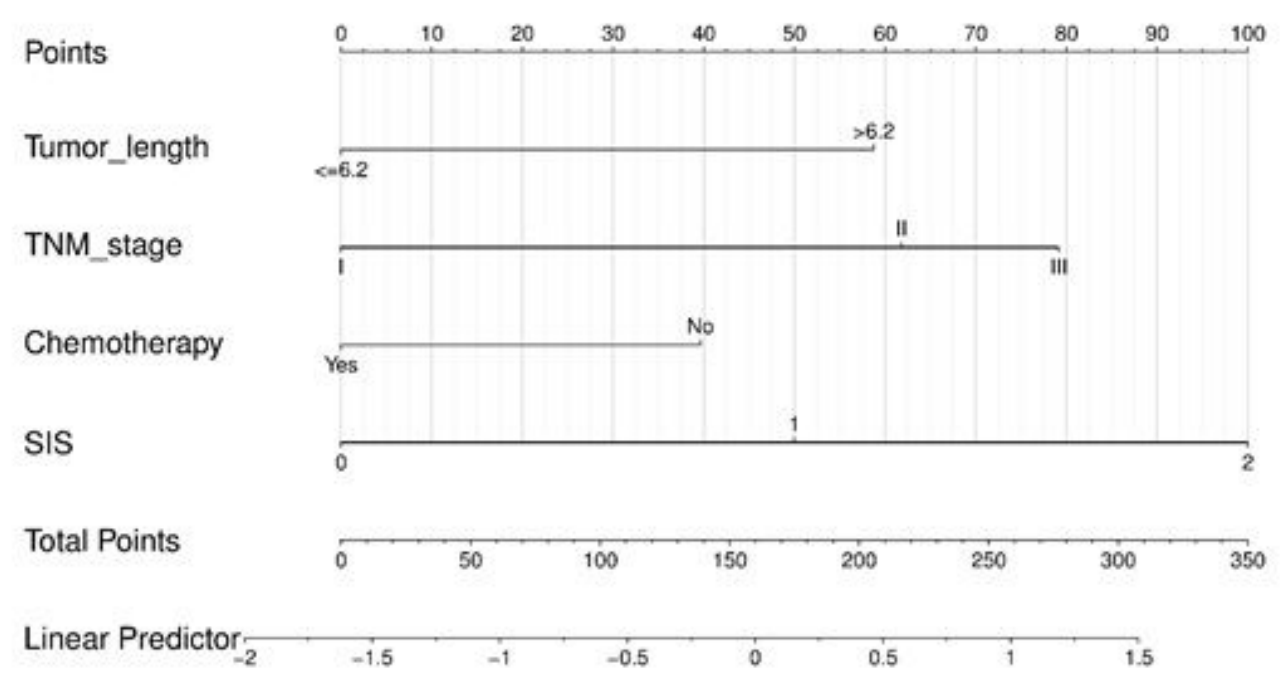

1-year PFS.

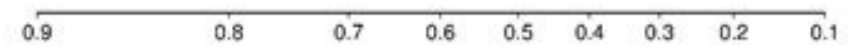

3-year PFS.

5-year PFS.
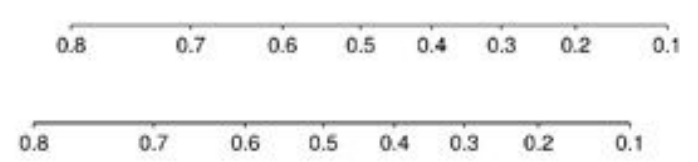

\section{Figure 5}

Nomogram predicting 1-, 3- and 5-year PFS probabilities of patients with ESCC. Each risk factor was assigned a point according to the nomogram. The exact values of each factor are Tumor length ( 0,59 points), TNM stage $(0,79$ points), Chemotherapy $(0,40$ points), SIS ( 0,100 points). The total points were obtained by adding the points of all risk factors. Then, the 1-, 3- and 5-year PFS could be predicted based on the total points. 
A

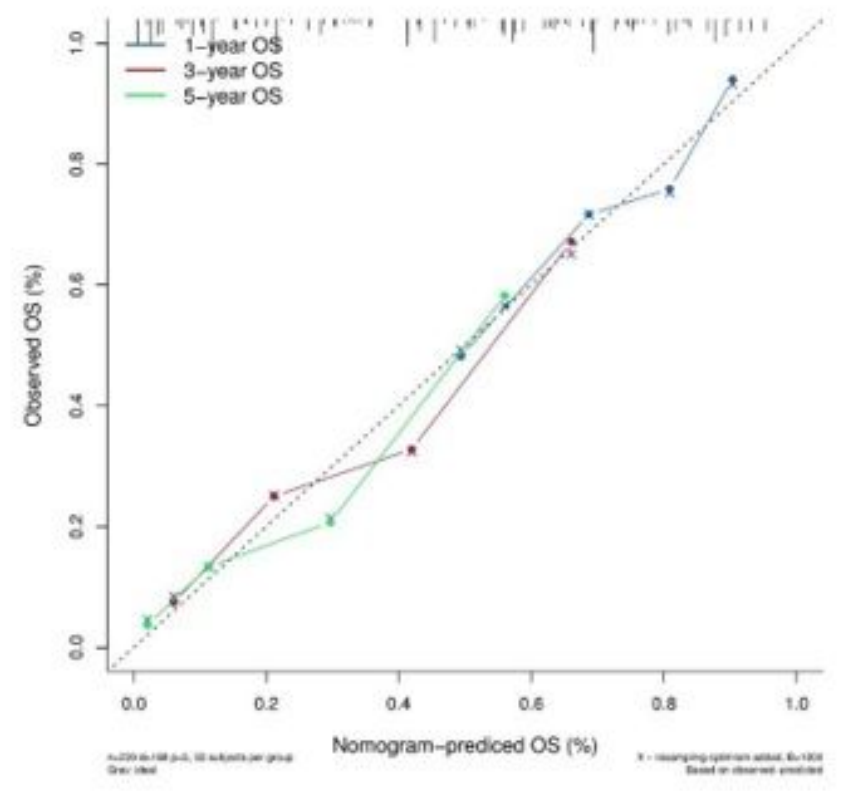

B

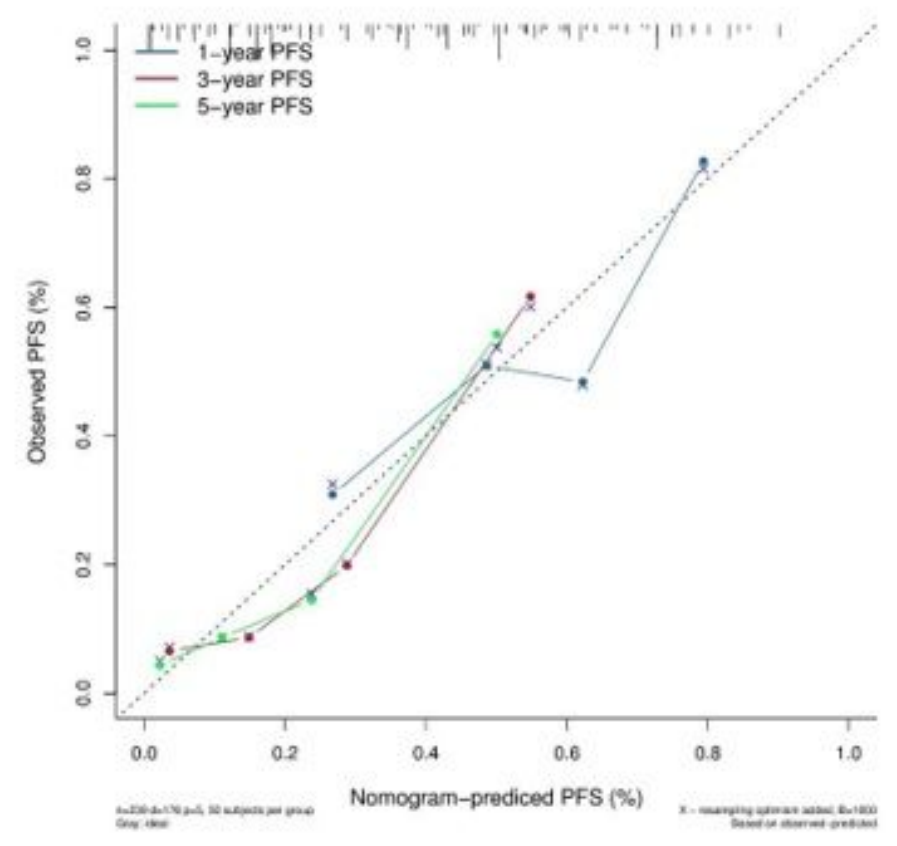

Figure 6

Calibration curve for predicting 1-,3- and 5-year OS (A), PFS (B). The nomogram-predicted probability of OS/PFS is plotted on the $x$-axis; the actual OS/PFS is plotted on the $y$-axis. 